University of Nebraska - Lincoln

DigitalCommons@University of Nebraska - Lincoln

Nutrition and Health Sciences -- Faculty

Publications

Nutrition and Health Sciences, Department of

2018

Sex- and Mode-specific Responses to Eccentric Muscle Fatigue

Ethan C. Hill

University of Nebraska-Lincoln, ethan.hill@unl.edu

Terry J. Housh

University of Nebraska-Lincoln, thoush1@unl.edu

Cory M. Smith

University of Nebraska - Lincoln, cmsmith7@utep.edu

Josh L. Keller

University of Nebraska - Lincoln, jkeller@unl.edu

Richard J. Schmidt

University of Nebraska - Lincoln, rschmidt1@unl.edu

See next page for additional authors

Follow this and additional works at: https://digitalcommons.unl.edu/nutritionfacpub

Part of the Human and Clinical Nutrition Commons, Molecular, Genetic, and Biochemical Nutrition Commons, and the Other Nutrition Commons

Hill, Ethan C.; Housh, Terry J.; Smith, Cory M.; Keller, Josh L.; Schmidt, Richard J.; and Johnson, Glen O., "Sex- and Mode-specific Responses to Eccentric Muscle Fatigue" (2018). Nutrition and Health Sciences -Faculty Publications. 154.

https://digitalcommons.unl.edu/nutritionfacpub/154

This Article is brought to you for free and open access by the Nutrition and Health Sciences, Department of at DigitalCommons@University of Nebraska - Lincoln. It has been accepted for inclusion in Nutrition and Health Sciences -- Faculty Publications by an authorized administrator of DigitalCommons@University of Nebraska Lincoln. 


\section{Authors}

Ethan C. Hill, Terry J. Housh, Cory M. Smith, Josh L. Keller, Richard J. Schmidt, and Glen O. Johnson 


\title{
Sex- and Mode-specific Responses to Eccentric Muscle Fatigue
}

\author{
Authors \\ Ethan C. Hill, Terry J. Housh, Cory M. Smith, Joshua L. Keller, Richard J. Schmidt, Glen O. Johnson
}

\author{
Affiliations \\ University of Nebraska-Lincoln, Nutrition and Health \\ Sciences, Lincoln, United States \\ Key words \\ blood flow, motor control, isokinetic, muscle fatigue, \\ gender, EMG, MMG
}

accepted 06.07.2018

\section{Bibliography}

DOI https://doi.org/10.1055/a-0664-0733

Published online: 11.9 .2018

Int J Sports Med 2018; 39: 893-901

(c) Georg Thieme Verlag KG Stuttgart · New York

ISSN 0172-4622

\section{Correspondence}

Mr. Ethan Cole Hill

Published online: 2018

Exercise \& Sport Science

Nutrition and Health Sciences

110 Ruth Leverton Hall

68505 Lincoln

United States

Tel.: + 1/262/2154 636, Fax: + 1/402/4722 690

ethan.hill@unl.edu

\begin{abstract}
The primary purpose of the present investigation was to examine sex-related differences as a result of fatiguing eccentric muscle actions on torque, muscle blood flow, electromyography, and mechanomyography. Eighteen men and 18 women performed peak torque trials prior to (pretest), immediately after (posttest), and 5-min after (recovery) completing 50 submaximal ( $60 \%$ of eccentric peak torque), eccentric, isokinetic $\left(180^{\circ} \cdot \mathrm{s}^{-1}\right)$ muscle actions of the elbow flexors. Electromyographic and mechanomyographic responses were simultaneously recorded from the biceps brachii muscle, and muscle blood flow was measured at pretest, posttest and recovery. There were sex- and mode-specific responses for torque, but there were no sex-specific muscle blood flow or neuromuscular responses. From pretest to posttest, torque decreased (80.0$88.2 \%$ of pretest) for both the men and women. At recovery concentric peak torque recovered to a greater extent in women $(95.0 \%$ of pretest) than men ( $88.0 \%$ of pretest), while eccentric peak torque recovered to a greater extent in men ( $88.9 \%$ of pretest) than women ( $86.9 \%$ of pretest). The sexspecific torque responses were not associated with different motor control strategies or differences in the occlusion of muscle blood flow between the men and women. For both men and women, eccentric fatiguing exercise is manifested similarly during isometric muscle actions, but not during eccentric and concentric muscle actions.
\end{abstract}

\section{Introduction}

It has been suggested that women are less susceptible than men to muscle fatigue as a result of concentric and isometric muscle actions [20,21]. Sex-related differences in muscle fatigue have been attributed to differences in muscle strength, the intensity of the fatiguing task, muscle blood flow, the velocity of contraction, and the muscle groups involved [20,21]. For example, Hunter and Enoka [24] reported that sex differences in the time to exhaustion during continuous isometric muscle actions at $20 \%$ of maximal voluntary isometric contraction (MVIC) were accounted for by differences in muscle strength. At higher intensities of exercise (70-80\% of MVIC), however, men and women experienced similar fatigueinduced decreases in performance (peak strength and/or time to exhaustion), while at lower intensities ( $20 \%$ of MVIC) of exercise men fatigued more rapidly than women [32]. In addition, Senefeld et al. [47] reported the men and women experienced similar reductions in MVIC torque following a 60-s maximal sustained isometric muscle action of the leg extensors. Furthermore, men experienced greater reductions in MVIC following maximal intermittent dorsiflexion muscle actions during non-ischemic conditions, but there were no sex differences when muscle blood flow was occluded [43]. Thus, it has been speculated that one of the underlying mechanisms of sex-specific fatigue responses is related to muscle blood flow [20,21]. Specifically, it is thought that greater absolute strength in men than women would result in greater occlusion of muscle blood flow and, consequently, increase the buildup of metabolites [20,21]. At higher intensities of exercise, it is believed that muscle blood flow occlusion is similar in men and women [20,21]. For example, when muscle blood flow was artificially occluded both men and women experienced similar reductions in maximal strength and time to exhaustion [43].

At a slow isokinetic velocity $\left(60^{\circ} \cdot \mathrm{s}^{-1}\right)$, women performed a greater number of elbow flexion muscle actions than men, when performed at $20 \%$ of MVIC to exhaustion [56]. At $20 \%$ of one-repetition maximum, there were no sex-related differences in MVIC or electromyographic (EMG) amplitude, when instructed to complete 
each forearm flexion muscle action as fast as possible [48]. Under the same conditions, however, men exhibited greater reductions in MVIC and EMG amplitude than women when instructed to complete each knee extension muscle action as fast as possible [48]. Furthermore, for the leg extensors men and women experienced similar reductions in maximal torque following 120 isokinetic $\left(60^{\circ} \cdot \mathrm{s}^{-1}\right)$ leg extension muscle actions performed at $20 \%$ of concentric peak torque (PT), but women recovered a greater extent than men over a 10-min period following exercise cessation. For the forearm flexors, however, there were no sex-related differences in the EMG or mechanomyographic (MMG) amplitude or mean power frequency responses, but sex-specific decreases in MVIC and concentric PT as a result of fatiguing isometric and concentric muscle actions performed at $65 \%$ of MVIC or concentric PT $[17,18]$. The lack of sex-specific EMG and MMG responses as a result of fatiguing concentric and isometric muscle actions of the forearm flexors suggested that the sex differences in the torque responses could not be attributed to differences in motor unit recruitment (EMG amplitude and MMG amplitude) or motor unit firing rates (EMG amplitude and MMG mean power frequency) $[17,18,48]$. Thus, for concentric and isometric muscle actions, sex differences depend largely on the characteristics of the fatiguing interventions and the muscle groups involved (i. e. upper versus lower body).

During eccentric muscle actions, however, the mechanisms mediating sex-specific fatigue responses may be different than those for isometric and concentric muscle actions [20]. In addition, unlike concentric and isometric muscle actions, women experienced greater fatigue-induced decreases in MVIC than men following submaximal [46] or maximal [49] eccentric muscle actions of the forearms flexors. For the leg extensors, however, there were no sex differences in maximal torque, power, voluntary activation, or resting twitch amplitude following 150 maximal isokinetic $\left(60^{\circ} \cdot s^{-1}\right)$ eccentric muscle actions [29]. Thus, it is possible that like fatiguing concentric and isometric muscle actions, potential sex differences following fatiguing eccentric muscle actions may be related to the characteristics of the fatiguing task or the muscle groups involved. Therefore, the primary purpose of the present investigation was to examine sex-related differences as a result of fatiguing eccentric muscle actions on torque, muscle blood flow, EMG, and MMG. Secondary purposes were to examine whether there were mode-specific decreases in eccentric PT, concentric PT, and MVIC as a result of fatiguing eccentric muscle actions. Based on previous investigations $[17,18,48]$, we hypothesized that there would be no sex-related differences in EMG or MMG, but sex-specific decreases in eccentric PT, concentric PT, and MVIC. Furthermore, the sex-related differences in torque would be associated with differences between the men and women in muscle blood flow responses, but there would be no mode-specific decreases in torque [21].

\section{Material and Methods}

\section{Subjects}

Twenty men and 20 women volunteered to participate in this investigation. However, two men and two women were unable to
- Table 1 Descriptive characteristics (mean \pm SD) for the men $(n=18)$ and women $(n=18)$.

\begin{tabular}{|c|c|c|}
\hline Variable & Men & Women \\
\hline Age (yrs) & $23.2 \pm 3.0$ & $22.3 \pm 1.7$ \\
\hline Body Weight (kg) * & $85.4 \pm 12.1$ & $64.1 \pm 8.3$ \\
\hline Height $(\mathrm{cm})^{*}$ & $179.6 \pm 8.2$ & $167.5 \pm 5.9$ \\
\hline $\begin{array}{l}\text { Resistance training } \\
\text { (hrs } \cdot \mathrm{wk}^{-} 1 \text { ) }\end{array}$ & $6.9 \pm 2.8$ & $6.3 \pm 3.5$ \\
\hline Body percentage fat (\%) ${ }^{*}$ & $11.1 \pm 4.2$ & $20.2 \pm 5.5$ \\
\hline Lean Body Mass (kg) ${ }^{*}$ & $75.9 \pm 10.0$ & $51.2 \pm 5.5$ \\
\hline
\end{tabular}

complete all testing procedures, and were therefore eliminated from all analyses ( $>$ Table 1 ). The subjects had no known cardiovascular, pulmonary, metabolic, muscular, and/or coronary heart disease, or regularly used prescription medication. In addition, at the time of testing all subjects had been actively participating in resistance training for at least the past six months. The subjects visited the laboratory on two occasions separated by at least 72-h and were instructed to avoid performing upper body exercise 48 -h prior to the testing visit. The subjects were rescheduled if they were experiencing any muscle soreness (score of two or greater on a 10point scale) as determined by a visual analog scale [31]. The study was approved by the University Institutional Review Board for Human Subjects, and the study was consistent with the International Journal of Sports Medicine's ethical standards [11]. All subjects completed a health history questionnaire, and signed a written informed consent prior to testing.

\section{Procedures}

Familiarization

The first laboratory visit consisted of an orientation session to familiarize the subjects with the testing protocols. During the orientation, body weight, height, and body percentage fat were determined for each subject. Body percentage fat was calculated from the seven-site skinfold equation of Jackson and Pollock [25]. Following the determination of subject characteristics, the subjects performed submaximal and maximal isometric muscle actions, as well as submaximal and maximal eccentric and concentric, isokinetic muscle actions of the elbow flexors at a velocity of $180^{\circ} \cdot \mathrm{s}^{-1}$. To familiarize the subjects with the fatiguing protocols, the subjects practiced performing eccentric, isokinetic muscle actions at a velocity of $180^{\circ} \cdot \mathrm{s}^{-1}$ and at an intensity that corresponded to $60 \%$ of their eccentric PT, which was visually tracked using real-time torque displayed on a computer monitor.

\section{Determination of eccentric PT, concentric PT, and MVIC}

During visit two, the subjects performed a warm-up consisting of three sets of 10 repetitions of reciprocal eccentric-concentric muscle actions of the dominant (based on throwing preference) elbow flexors with 1 -min of rest between sets. Each repetition was performed at a velocity of $180^{\circ} \cdot \mathrm{s}^{-} 1$ on a calibrated isokinetic dynamometer (Cybex 6000) at approximately $50 \%$ effort. Following the warmup, the subjects rested for 5 -min and then randomly performed two pretest maximal eccentric PT $\left(180^{\circ} \cdot \mathrm{s}^{-1} 1\right)$, concentric PT $\left(180^{\circ} \cdot \mathrm{s}^{-} 1\right)$, and MVIC trials. The eccentric and concentric mus- 
cle actions were performed through a $90^{\circ}$ range of motion (0-90 of elbow flexion, where $0^{\circ}$ corresponds to full extension at the elbow). The isometric muscle actions were performed at a joint angle of $45^{\circ}$ and each MVIC trial was sustained for 3-s [26]. Peak torque and MVIC trials were also performed immediately (posttest) and 5-min after (recovery) completing the fatiguing protocol. The posttest and recovery trials were performed in the same order using the same procedures as the pretest trials.

\section{Fatiguing protocol at $60 \%$ of eccentric PT}

Following the determination of the pretest eccentric PT, concentric PT, and MVIC, the subjects performed 50 eccentric-only muscle actions of the elbow flexors at $60 \%$ of their pretest eccentric PT. Real-time torque was displayed on a computer monitor and each eccentric muscle action was performed at a velocity of $180^{\circ} \cdot \mathrm{s}^{-1}$ and was followed by a passive concentric muscle action that was assisted by the investigator.

\section{Electromyographic and mechanomyographic measurements}

During visit two, pre-gelled surface electrodes (Ag/AgCl, AccuSensor, Lynn Medical, Wixom, MI, USA) were placed in a bipolar arrangement ( $30 \mathrm{~mm}$ center-to-center) on the dominant arm over the biceps brachii according to the recommendations of Barbero et al. [3]. The reference electrode was placed over the acromion process, and prior to each electrode placement, the skin was shaved, carefully abraded, and cleaned with alcohol. The MMG signals from the biceps brachii were detected using an accelerometer (Entran EGAS FT 10, dimensions: $1.0 \times 1.0 \times 0.5 \mathrm{~cm}$, mass: $1.0 \mathrm{~g}$ ) that was placed between the proximal and distal EMG electrodes of the bipolar arrangement using double-sided adhesive tape. The raw EMG and MMG signals were digitized at $2,000 \mathrm{~Hz}$ with a 32-bit analog-to-digital converter (Model MP100, Biopac Systems, Inc.) and stored in a personal computer (ATIV Book 9 Intel Core i7 Samsung Inc., Dallas, TX) for subsequent analyses. The EMG signals were amplified (gain: x 1,000) using differential amplifiers (EMG 100, Biopac Systems, Inc., Santa Barbara, CA). The EMG and MMG signals were digitally bandpass filtered (fourth-order Butterworth, zero-phase shift) at $10-500 \mathrm{~Hz}$ and $5-100 \mathrm{~Hz}$, respectively. All signal processing was performed in LabVIEW. The amplitude of the EMG ( $\mu \mathrm{V}$ root-mean-square, $\mu \mathrm{Vrms}$ ) and MMG $\left(\mathrm{m} \cdot \mathrm{s}^{-2}\right)$ signals, as well as the mean power frequency $(\mathrm{Hz})$ values for the eccentric and concentric contractions were calculated from $30-60^{\circ}$ of flexion at the elbow $\left(0^{\circ}\right.$ corresponds to full extension at the elbow). Thus, signal epochs of 0.17-s (333 data points) were used to calculate the EMG and MMG values associated with the eccentric and concentric muscle actions. Similarly, the EMG and MMG values during the MVIC muscle actions were calculated for a time period that corresponded to 1-s (2,000 data points) over the middle $1 / 3$ of the muscle action. These portions of the signals were selected to avoid the acceleration and deceleration phases that are typical of isokinetic dynamometers [5] and to avoid the initial gross lateral movement that occurs at the onset of a muscle action [36].

\section{Ultrasound measurements}

Muscle blood flow was assessed via ultrasound prior to, immediately after (within $57 \pm 8 \mathrm{~s}$ ), and 5-min (298 $\pm 3 \mathrm{~s}$ ) after completing the fatiguing protocol. Ultrasound images of the dominant elbow flexors were obtained using a portable brightness mode ultrasound imaging device (GE Logiq e, USA) and a multi-frequency linear-array probe (12L-Rs; 5-13 MHz; $38.4 \mathrm{~mm}$ field-of-view). Blood flow was assessed from the brachial artery proximal to the antecubital fossa [7] using Pulsed Wave Doppler at a repetition frequency of $10 \mathrm{MHz}$ and once identified, the location was marked with a permanent marker. All blood flow measurements were assessed at an insonation angle of $60^{\circ}$ to the brachial artery collected over a period of three cardiac cycles [37, 38] and derived based on previous recommendations [8]. All measurements were taken while the subjects were lying in the supine position on the isokinetic dynamometer with both their arms and legs supported. Great care was taken to ensure that consistent, minimal pressure was applied with the probe to limit compression of the artery.

\section{Data analysis}

Normalization

To allow for the comparison between sexes and modes, the neuromuscular parameters (EMG amplitude, EMG mean power frequency, MMG amplitude, MMG mean power frequency) were normalized to the pretest MVIC neuromuscular responses. For all other analyses including eccentric PT, concentric PT, MVIC, and muscle blood flow, absolute values were used.

\section{Statistical analyses}

A 2 (Sex [men, women]) $\times 3$ (Time [pretest, posttest, recovery] $) \times 3$ (Mode [eccentric PT, concentric PT, MVIC]) mixed factorial ANOVA was used to analyze the torque responses as a result of the fatiguing protocol. Muscle blood flow was examined using a 2 (Sex [men, women]) $\times 3$ (Time [pretest, posttest, recovery]) mixed factorial ANOVA and the neuromuscular responses during the eccentric PT, concentric PT, and MVIC trials were examined using four (EMG amplitude, EMG mean power frequency, MMG amplitude, and MMG mean power frequency) separate 2 (Sex [men, women]) $\times 3$ (Time [pretest, posttest, recovery]) $\times 3$ (Mode [eccentric PT, concentric PT, $M V I C]$ ) mixed factorial ANOVAs. Significant interactions were decomposed with follow-up repeated measures ANOVAs and Bonferonni-corrected independent or dependent samples $t$-tests. Greenhouse-Geisser corrections were applied when sphericity was not met according to Maulchy's Test of Sphericity and partial eta squared effect sizes $\left(\eta_{p}^{2}\right)$ were calculated for each ANOVA. All statistical analyses were performed using IBM SPSS v. 21 (Armonk, NY) and an alpha of $p \leq 0.05$ considered statistically significant for all comparisons.

\section{Results}

\section{Torque responses}

There was no significant 3-way interaction (Sex $\times$ Time $\times$ Mode), but there were three significant 2-way (Sex $\times$ Time, Sex $\times$ Mode, Mode $\times$ Time) interactions for torque ( $\vee$ Table 2$)$. As a result, the 3-way ANOVA was decomposed into separate Time $\times$ Mode repeated measures ANOVAs by Sex.

For the men, there was a significant two-way Time $\times$ Mode interaction and significant simple main effects ( $\triangleright$ Table 2 ). Followup analyses indicated that there were decreases from pretest to posttest for eccentric PT (decreased to $80.0 \%$ of pretest), concen- 
- Table 2 Displays the effects for each variable with the corresponding $p$-value and partial eta squared effect size. In the event of an interaction(s), only the main effect(s) not involved in the interaction(s) were provided.

\begin{tabular}{|c|c|c|c|}
\hline Variable & Effect & p-Value & $\begin{array}{l}\text { Partial Eta } \\
\text { Squared }\end{array}$ \\
\hline \multirow[t]{4}{*}{ Torque } & Sex $\times$ Time $\times$ Mode & 0.503 & 0.026 \\
\hline & Sex $\times$ Time & $<0.001$ & 0.775 \\
\hline & Sex $\times$ Mode & $<0.001$ & 0.701 \\
\hline & Mode $\times$ Time & $<0.001$ & 0.861 \\
\hline Muscle Blood Flow & Sex $\times$ Time & 0.003 & 0.170 \\
\hline \multirow[t]{5}{*}{ EMG Amplitude } & Sex $\times$ Time $\times$ Mode & 0.932 & 0.007 \\
\hline & Sex $\times$ Time & 0.782 & 0.008 \\
\hline & Sex $\times$ Mode & 0.055 & 0.087 \\
\hline & Mode $\times$ Time & 0.002 & 0.263 \\
\hline & Sex & 0.498 & 0.014 \\
\hline \multirow{7}{*}{$\begin{array}{l}\text { EMG Mean Power } \\
\text { Frequency }\end{array}$} & Sex $\times$ Time $\times$ Mode & 0.768 & 0.014 \\
\hline & Sex $\times$ Time & 0.707 & 0.011 \\
\hline & Sex $\times$ Mode & 0.629 & 0.014 \\
\hline & Mode $\times$ Time & 0.230 & 0.043 \\
\hline & Sex & 0.420 & 0.020 \\
\hline & Mode & 0.013 & 0.127 \\
\hline & Time & $<0.001$ & 0.329 \\
\hline \multirow[t]{7}{*}{ MMG Amplitude } & Sex $\times$ Time $\times$ Mode & 0.557 & 0.023 \\
\hline & Sex $\times$ Time & 0.488 & 0.022 \\
\hline & Sex $\times$ Mode & 0.148 & 0.058 \\
\hline & Mode $\times$ Time & 0.121 & 0.055 \\
\hline & Sex & 0.104 & 0.081 \\
\hline & Mode & $<0.001$ & 0.456 \\
\hline & Time & 0.998 & 0.001 \\
\hline \multirow{7}{*}{$\begin{array}{l}\text { MMG Mean Power } \\
\text { Frequency }\end{array}$} & Sex $\times$ Time $\times$ Mode & 0.459 & 0.028 \\
\hline & Sex $\times$ Time & 0.137 & 0.060 \\
\hline & Sex $\times$ Mode & 0.556 & 0.018 \\
\hline & Mode ×Time & 0.665 & 0.018 \\
\hline & Sex & 0.109 & 0.078 \\
\hline & Mode & 0.007 & 0.143 \\
\hline & Time & 0.350 & 0.032 \\
\hline
\end{tabular}

tric PT (80.5\%), and MVIC (87.1\%) that returned to pretest levels at recovery for MVIC $(96.0 \%)$, but only partially recovered for eccentric PT (88.9\%) and concentric PT (88.0\%) ( $\triangleright$ Fig. 1). At pretest, eccentric PT $(71.3 \mathrm{Nm})$ was greater than MVIC $(59.5 \mathrm{Nm})$ and concentric PT (44.2 Nm), and MVIC was greater than concentric PT. At posttest and recovery, however, both eccentric PT $(57.0 \mathrm{Nm}$ and $63.3 \mathrm{Nm}$, respectively) and MVIC ( $51.8 \mathrm{Nm}$ and $57.2 \mathrm{Nm}$, respectively) were greater than concentric PT ( $35.5 \mathrm{Nm}$ and $38.8 \mathrm{Nm}$, respectively), but eccentric PT was not greater than MVIC at posttest or recovery.

For the women, there was a significant two-way Time $\times$ Mode interaction and significant simple main effects ( $\vee$ Table 2 ). Followup analyses indicated that there were decreases from pretest to posttest for eccentric PT (decreased to $82.5 \%$ of pretest), concentric PT $(86.1 \%)$, and MVIC $(88.2 \%)$ that returned to pretest levels at recovery for concentric PT (95.0\%) and MVIC (99.6\%), but re- mained depressed for eccentric PT (86.9\%) ( $\triangleright$ Fig. 1). At pretest, posttest, and recovery, eccentric PT $(41.4 \mathrm{Nm}, 34.2 \mathrm{Nm}$, and $36.0 \mathrm{Nm}$, respectively) was greater than MVIC $(29.1 \mathrm{Nm}, 24.8 \mathrm{Nm}$, and $29.0 \mathrm{Nm}$, respectively), and MVIC was greater than concentric PT (23.8 Nm, $20.5 \mathrm{Nm}$, and $22.6 \mathrm{Nm}$, respectively) at all time points.

\section{Muscle blood flow responses}

There was a significant Sex $\times$ Time interaction for muscle blood flow ( $\triangleright$ Table 2). Analyses for simple main effects, however, indicated that the men and women exhibited very similar fatigue-related patterns of responses for muscle blood flow. Specifically, muscle blood flow increased from pretest to posttest, and had partially returned to pretest levels at recovery for both the men and women ( $\vee$ Fig. 2). At pretest, posttest, and recovery, however, muscle blood flow $\left(\mathrm{mL} \cdot \mathrm{min}^{-1} 1\right)$ was greater for the men than the women.

\section{Neuromuscular responses}

EMG amplitude

There was no significant 3 -way interaction (Sex $\times$ Time $\times$ Mode), but there was a significant Time $\times$ Mode (collapsed across Sex) interaction for EMG amplitude, but no main effect for Sex ( $\bullet$ Table 2). Follow-up one-way ANOVAs for Mode and Time indicated that there were decreases from pretest and posttest to recovery for MVIC (pretest and posttest > recovery), and a decrease from posttest to recovery for concentric PT (posttest > recovery), while there were no changes during the eccentric PT muscle actions (pretest = posttest $=$ recovery) $(\triangleright$ Fig. 3$)$. At pretest, EMG amplitude was greater during the concentric PT than eccentric PT muscle actions (concentric PT > eccentric PT). At posttest, EMG amplitude was greater during the concentric PT and MVIC than eccentric PT muscle actions (concentric PT and MVIC > eccentric PT). At recovery, EMG amplitude was greater during the concentric PT than MVIC muscle actions (concentric PT > MVIC). Collectively, during the eccentric PT muscle actions, EMG amplitude was lower and remained unchanged at all time points. On the contrary, EMG amplitude was higher during the concentric PT muscle actions, but EMG amplitude decreased during the concentric PT and MVIC muscle actions.

\section{EMG mean power frequency}

There was no significant 3-way (Sex $\times$ Time $\times$ Mode), or 2-way (Sex $\times$ Time, Sex $\times$ Mode, Mode $\times$ Time) interactions for EMG mean power frequency ( $\triangleright$ Table 2). There was, however, main effects for Mode (collapsed across Sex and Time; eccentric PT > MVIC > concentric PT) and Time (collapsed across Sex and Mode; recovery > pretest > posttest). Thus, EMG mean power frequency was greater during the eccentric PT muscle actions. For all three modes and both sexes, EMG mean power frequency decreased from pretest to posttest, but increased from posttest to recovery and was greater at recovery than at pretest.

\section{MMG amplitude}

There was no significant 3-way (Sex $\times$ Time $\times$ Mode), or 2-way (Sex $\times$ Time, Sex $\times$ Mode, Mode $\times$ Time) interactions for MMG amplitude ( $\triangleright$ Table 2 ). There was, however, a main effect for Mode (collapsed across Sex and Time) that was greater during the eccentric PT and concentric PT than MVIC muscle actions (eccentric PT and concentric $\mathrm{PT}>\mathrm{MVIC}$ ). 


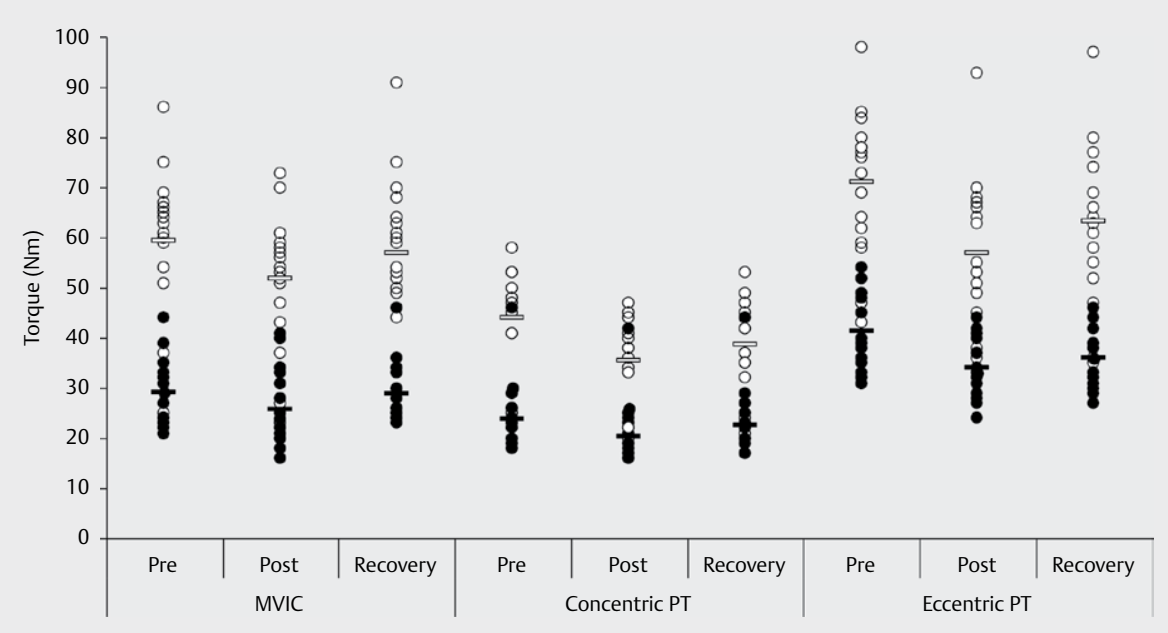

Fig. 1 The individual (circles) and mean (horizontal lines) pretest, posttest, and recovery responses for eccentric peak torque (PT), concentric PT, and maximal voluntary isometric contraction (MVIC) for the men (open circles, open line) and women (filled circles, filled line).

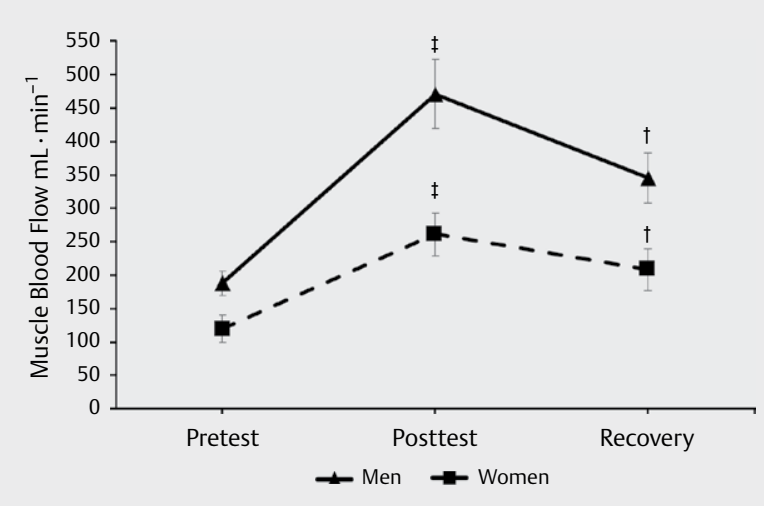

- Fig. 2 The pretest, posttest, and recovery muscle blood flow responses (mean $\pm \mathrm{SE}$ ) for the men and women. There were significant $(p<0.05)$ increases from pretest to posttest that partially returned to pretest levels at recovery ${ }^{\dagger}$ for both the men and women. There was no difference in the relative increases in muscle blood flow from pretest to posttest or the recovery responses between the men and women.

\section{MMG mean power frequency}

There was no significant 3-way (Sex $\times$ Time $\times$ Mode), or 2-way (Sex $\times$ Time, Sex $\times$ Mode, Mode $\times$ Time) interactions for MMG mean power frequency ( $\triangleright$ Table 2). There was, however, a main effect for Mode (collapsed across Sex and Time) that was greater during the eccentric PT and concentric PT than MVIC muscle actions (eccentric PT and concentric PT > MVIC).

\section{Discussion}

\section{Torque responses}

In the present study, there were sex-specific torque responses that resulted in differences in the magnitudes of recovery. Specifically,

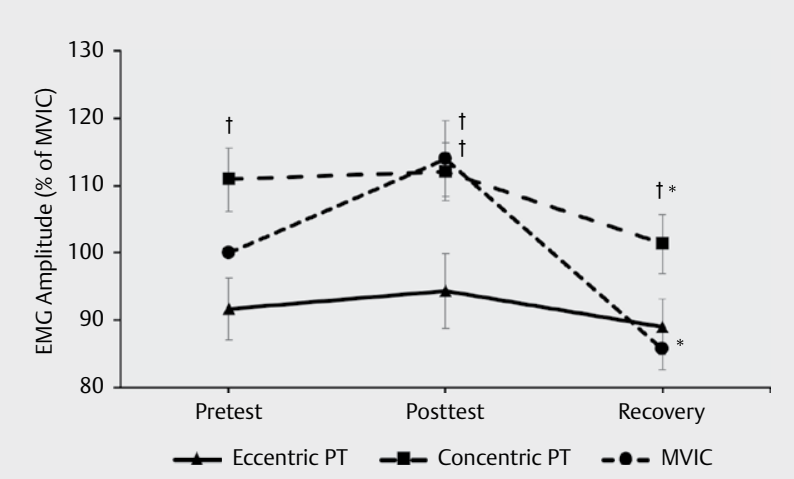

- Fig 3 The electromyographic (EMG) amplitude responses (mean $\pm \mathrm{SE}$ ) during the eccentric peak torque (PT), concentric PT, and maximal voluntary isometric contraction (MVIC) muscle actions (collapsed across Sex) at pretest, posttest, and recovery. There were significant $(p<0.05)$ decreases from pretest and posttest to recovery * during the concentric PT and MVIC muscle actions. At all time points, EMG amplitude was greater ${ }^{\dagger}$ during the concentric PT than eccentric PT muscle actions and at posttest EMG amplitude was greater ${ }^{\dagger}$ during the MVIC than eccentric PT muscle actions.

for both the men and women, there were decreases from pretest to posttest for eccentric PT, concentric PT, and MVIC. At recovery, $\mathrm{MVIC}$ returned to pretest levels for both the men and women. There was, however, sex-specific recovery responses for eccentric PT that partially returned to pretest levels for the men, but remained depressed for the women. In addition, concentric PT returned to pretest levels for the women, but only partially returned to pretest levels for the men. Thus, following the fatiguing eccentric muscle actions, eccentric PT recovered to a greater in extent in men, while concentric PT recovered to a greater extent in women.

The present findings were consistent with previous investigations $[17,18]$ that have examined sex-specific responses as a result of fatiguing exercise. Specifically, fatiguing isometric or concentric muscle actions performed at $65 \%$ of MVIC or concentric PT result- 
ed in sex-specific decreases in MVIC and concentric PT [17, 18]. The current findings, in conjunction with previous investigations $[17,18]$, indicated that performing dynamic fatiguing muscle actions at intensities $\leq 65 \%$ of maximum torque resulted in sex-related torque responses. At higher intensities of exercise, however, it has been reported that men and women fatigued similarly [21]. For example, there were no sex-related differences in the posttest decreases in MVIC or concentric PT as a result of fatiguing concentric or isometric muscle actions performed at $80-90 \%$ of MVIC or concentric PT $[32,40,41,56]$. Thus, like fatiguing concentric and isometric muscle actions, there may exist sex-specific torque responses to fatiguing eccentric muscle actions that are dependent upon the intensity of the fatiguing exercise.

The sex-specific recovery responses in the present study were partially consistent with previous investigations [18, 22, 40,41, 43]. For example, like the present findings for eccentric muscle actions, concentric PT and MVIC recovered to a greater extent in women than men after 5 - or 10-min of recovery following fatiguing isometric and concentric interventions performed at intensities of $65-100 \%$ of MVIC or concentric PT $[18,22,43]$. In addition, there were no sexspecific responses for MVIC torque or power following 150 maximal eccentric muscle actions of the leg extensors performed at $\left.60^{\circ} \cdot \mathrm{s}^{-1} 129\right]$. After 24 -h of recovery, however, muscle power assessed during a concentric muscle action performed at $60 \%$ of MVIC recovered to a greater extent in men than women, but there were no differences in power assessed from 10-50\% of MVIC [29]. On the contrary, MVIC recovered to a greater extent in men than women following 150 maximal eccentric muscle actions of the dorsiflexors [41]. There were, however, no sex-specific recovery responses following 150 eccentric muscle actions of the dorsiflexors at $80 \%$ of MVIC [40]. Therefore, for the dorsiflexors there are conflicting results regarding sex-specific responses to high-intensity fatiguing eccentric muscle actions. In the present study, for the elbow flexors concentric PT recovered to a greater extent for the women, while eccentric PT recovered to a greater extent for the men. Together, the present findings and those of Lee et al. [29] indicated that fatiguing eccentric muscle actions of the forearm flexors and leg extensors resulted in sex-specific torque responses that were manifested during concentric muscle actions, but there were no sex-specific responses during isometric muscle actions at any time points.

For both the men and women, MVIC torque returned to pretest levels at recovery which may have been related to a shift in the optimal angle of force production. For example, Prasartwuth et al. [42] reported that the optimal joint angle for MVIC increased $16.7^{\circ}$ to a longer muscle length following fatiguing eccentric muscle actions of the elbow flexors. In addition, eccentric muscle actions have been applied in clinical and athletic settings to induce shifts in the optimal angle of force production as a potential protective mechanism to reduce the prevalence of sport-related injuries [6]. On the contrary, Lee et al. [29] reported no change in the optimal angle of force production following 150 maximal eccentric muscle actions. The authors [29] suggested that the lack of change in the optimal angle of force production may have been due to prior exercise training experience. Thus, it is possible that the fatiguing eccentric muscle actions in the preset study induced a shift in the optimal angle of force production, and it is also possible that fatigueinduced decreases in torque as a result of fatiguing eccentric muscle actions were manifested differently between isometric versus dynamic muscle actions.

\section{Muscle blood flow}

There was greater absolute muscle blood flow in men compared to women at pretest, posttest, and recovery, but there was no difference in the fatigue-related patterns of changes in muscle blood flow between the men and women tested. Specifically, muscle blood flow increased from pretest to posttest for both the men and women, and partially returned to pretest levels at recovery. These findings were not consistent with previous investigations [30, 39] that have reported sex-related differences in muscle blood flow as a result of fatiguing concentric or isometric interventions. That is, compared to men, women exhibited greater relative increases in muscle blood flow as a result of maximal effort leg extension muscle actions performed to exhaustion and following 3-min of blood flow occlusion of the brachial artery [30,39]. The fatigue-related change in muscle blood flow in the present study indicated that fatiguing eccentric muscle actions at $60 \%$ of eccentric PT resulted in the same patterns of response from pretest to posttest and posttest to recovery in men and women.

Increases in muscle blood flow as a result of exercise have been used to make inferences regarding sex-related differences in the magnitude of blood flow occlusion and exercise-induced increases in muscle blood flow $[21,23,24]$. For example, mechanical compression of the arteries increases with muscle strength and/or exercise intensity $[14,24,27]$. Thus, muscle blood flow may be affected to a greater extent in men than women due to differences in absolute muscle strength [21]. In addition, strength-related differences between men and women may contribute to an earlier onset of muscle blood flow occlusion at lower intensities of exercise in men than women $[21,23,24]$. The extent to which muscle blood flow contributes to muscle fatigue in men versus women during dynamic exercise, however, remains unclear [24, 39]. As a result of isolated eccentric or concentric fatiguing interventions, during the passive return of the lever arm or external load there is a removal of tension from the working muscle that may facilitate a decrease in the mechanical compression of the arteries. Thus, it is possible that dynamic muscle actions do not result in the same magnitude of blood flow occlusion as isometric muscle actions. In addition, eccentric muscle actions are less metabolically demanding than concentric and isometric muscle actions and, therefore, may stimulate smaller changes in muscle blood flow during fatiguing exercise. Consistent with this hypothesis, the findings of the present study indicated that muscle blood flow was not associated with sex-specific torque responses as a result of fatiguing eccentric muscle actions and muscle blood flow had partially returned to pretest levels after 5 -min of recovery. Therefore, the mechanisms underlying sex-specific decreases in torque as a result of fatiguing eccentric muscle actions may be different than fatiguing concentric and isometric muscle actions.

\section{Neuromuscular responses}

There were no sex-specific patterns of changes for any of the neuromuscular parameters and there were no changes from pretest to posttest for EMG amplitude, MMG amplitude, or MMG mean power frequency. These findings suggested that motor unit activation 
(EMG amplitude), motor unit recruitment (MMG amplitude), and motor unit firing rate (MMG mean power frequency) remained unchanged from pretest to posttest. There were, however, decreases for EMG mean power frequency from pretest to posttest that were consistent with the decreases in eccentric PT, concentric PT, and MVIC as a result of the fatiguing eccentric intervention. The present findings were consistent with those of Lee et al. [29] who reported no sex-related differences in voluntary activation or resting twitch amplitude following maximal fatiguing eccentric muscle actions of the leg extensors. After 48-hr of recovery, resting twitch amplitude returned to pretest levels, but voluntary activation remained depressed. Thus, the authors [29] suggested that both central and peripheral mechanisms mediated the fatigue-induced decreases in strength and power immediately and 24-h after fatiguing eccentric muscle actions. At 48-h into recovery, the decreases in strength and power were attributed to central mechanisms as indicated by voluntary activation that was still depressed [29]. Together, the present findings in conjunction with those of Lee et al. [29] indicated that fatiguing eccentric muscle actions do not result in sex-specific neuromuscular responses.

The fatigue-induced decreases in EMG mean power frequency from pretest to posttest for all three modes of muscle actions supported the fatiguing nature of the 50 repeated, submaximal, eccentric muscle actions. Specifically, fatiguing exercise has been associated with decreases in EMG mean power frequency that may reflect the effect of the accumulation of metabolic byproducts on muscle fiber action potential conduction velocity $[15,16,18]$. The increases in EMG mean power frequency at recovery may have reflected the clearance of metabolic byproducts or increases in muscle temperature [1]. In the present study, it is possible that some of the elastic recoil energy associated with the eccentric muscle actions may have been dissipated as heat [10] and/or exercise-induced increases in muscle blood flow increased muscle temperature [1].

During the eccentric PT muscle actions, EMG amplitude was lower and EMG mean power frequency was greater than during the concentric PT and MVIC muscle actions. These findings were consistent with previous investigations and may reflect selective recruitment $[33,34,50]$ and/or increased inhibitory feedback during eccentric muscle actions [2,52]. It has been theorized that lower muscle activation and faster action potential conduction velocity responses during eccentric muscle actions may reflect an improved distribution of mechanical stress on a fewer number of motor units, specifically fast-twitch motor units $[10,13]$. In addition, increased inhibitory feedback due to high contractile forces associated with eccentric muscle actions may cause a net decrease in muscle activation $[10,13]$. Thus, it is possible that the lower EMG amplitude, but greater EMG mean power frequency responses during the eccentric PT muscle actions reflected mode-specific motor control strategies to modulate torque production. Furthermore, MMG amplitude and MMG mean power frequency values were greater at pretest, posttest, and recovery during the eccentric PT and concentric PT than MVIC muscle actions. These findings were also consistent with previous investigations $[15,16]$ that have examined MMG responses for dynamic versus static muscle actions. In conjunction with previous investigations $[15,16]$, the findings of the present study suggested that dynamic muscle actions may have resulted in greater intracellular and extracellular fluid turbulence, which caused greater muscle perturbations and increased the MMG values [4].

Collectively, the results of the present study indicated that there were no sex-specific patterns of changes for the EMG or MMG responses. There were, however, decreases in EMG mean power frequency from pretest to posttest for all modes of muscle actions that supported the fatiguing nature of the exercise intervention and may have reflected the buildup of metabolic byproducts. The lower EMG amplitude values and greater EMG mean power frequency values during the eccentric PT muscle actions may have reflected mode-specific differences in the motor control strategies that modulate torque production. The greater MMG amplitude and MMG mean power frequency responses during the eccentric PT and concentric PT than MVIC muscle actions were likely due to the dynamic versus static characteristics of the muscle actions.

\section{Summary}

The findings of the present study indicated that there were sex- and mode-specific responses for torque. Specifically, the magnitude of torque recovery was greater for the women during the concentric PT muscle actions, but greater for the men during the eccentric PT muscle actions. There were, however, no sex-related differences for muscle blood flow or any of the neuromuscular parameters. Muscle blood flow increased from pretest to posttest and partially returned to pretest levels at recovery. In general, EMG amplitude was lower and EMG mean power frequency was higher, respectively, during the eccentric PT than the concentric PT and MVIC muscle actions. In addition, MMG amplitude and MMG mean power frequency values were greater at pretest, posttest, and recovery during the eccentric PT and concentric PT than MVIC muscle actions that were likely due to the dynamic versus static characteristics of the muscle actions. Collectively, the findings of the present study indicated that there were sex- and mode-specific responses for torque, but there were no sex-specific muscle blood flow or neuromuscular responses as a result of fatiguing eccentric muscle actions performed at $60 \%$ of eccentric PT and at a velocity of $180^{\circ} \cdot \mathrm{s}^{-1}$

\section{Limitations}

In the present study, the women were not asked to provide information regarding their phase within the ovarian cycle or whether they were using a contraceptive. Muscle blood flow and muscle strength, however, may be affected by different phases within an ovarian cycle. For example, the increases in flow-mediated dilation of the brachial artery were greater during the follicular $(18.2 \pm 0.8 \%)$ and luteal $(17.5 \pm 0.7 \%)$ phases than the menstrual phase $(11.2 \pm 0.6 \%)$ [12]. In a similar study, the increases in flow-mediated dilation of the brachial artery were enhanced during the early follicular $(8.8 \pm 0.6 \%)$ and late follicular $(10.0 \pm 0.7 \%)$ phases than in the early luteal $(4.2 \pm 0.6)$ and late luteal $(2.7 \pm 0.4 \%)$ phases [55]. Despite differences in flow-mediated dilation in previous studies $[12,55]$, there were no significant differences in mean blood flow across phases of the ovarian cycle [28]. Furthermore, using testretest reliability values, we [19] recently demonstrated that changes of $80 \%$ or more in baseline muscle blood flow for women would be required to identify a "real" [53] change. Thus, our previous findings [19] indicated that the magnitude of typical changes in mus- 
cle blood flow across phases of the ovarian cycle $[12,55]$ represented approximately $1-2 \%$ of the exercise-induced changes in the present study and, therefore, would likely not be of sufficient magnitude to affect the responses in the present study.

In regards to the effects of the ovarian cycle on muscle strength, previous investigations $[51,54]$ have reported that strength was greater during the follicular phase, while other investigations $[9,44]$ reported that strength was greater during the luteal phase. For women taking contraceptives, however, there appears to be no effect on strength performance [35, 45]. In the present study, all subjects completed the testing visits within $72-96 \mathrm{~h}$ and the ICC values for muscle blood flow and strength across the testing visits were $R=0.888$ and $R=0.961$, respectively. These findings in conjunction with previous investigations $[12,45,55]$, suggested that it is not likely that the ovarian cycle affected the muscle blood flow or strength responses in the present study.

\section{Acknowledgements}

This research did not receive any specific grant from funding agencies in the public, commercial, or not-for-profit sectors.

\section{Conflict of Interest}

No conflict of interest has been declared by the author(s).

\section{References}

[1] Arendt-Nielsen L, Mills KR, Forster A. Changes in muscle fiber conduction velocity, mean power frequency, and mean EMG voltage during prolonged submaximal contractions. Muscle Nerve 1989; 12: 493-497

[2] Babault N, Desbrosses K, Fabre M-S, Michaut A, Pousson M. Neuromuscular fatigue development during maximal concentric and isometric knee extensions. J Appl Physiol 2006; 100: 780-785

[3] Barbero M, Merletti R, Rainoldi A. Atlas of Muscle Innervation Zones Understanding Surface Electromyography and its Applications. New York, NY: Springer; 2012

[4] Beck TW, Housh TJ, Cramer JT, Weir JP, Johnson GO, Coburn JW, Malek $\mathrm{MH}$, Mielke M. Mechanomyographic amplitude and frequency responses during dynamic muscle actions: A comprehensive review. Biomed Engin 2005; 4: 67

[5] Brown LE, Whitehurst M, Gilbert R, Buchalter DN. The effect of velocity and gender on load range during knee extension and flexion exercise on an isokinetic device. J Orthop Sports Phys Ther 1995; 21: 107-112

[6] Brughelli M, Cronin J. Altering the length-tension relationship with eccentric exercise: Implications for performance and injury. Sports Med 2007; 37: 807-826

[7] Corretti MC, Anderson TJ, Benjamin EJ, Celermajer D, Charbonneau F, Creager MA, Deanfield J, Drexler H, Gerhard-Herman M, Herrington D, Vallance P, Vita J, Vogel R. Guidelines for the ultrasound assessment of endothelial-dependent flow-mediated vasodilation of the brachial artery: A report of the International Brachial Artery Reactivity Task Force. J Am Coll Cardiol 2002; 39: 257-265

[8] Gassner M, Killu K, Bauman Z, Coba V, Rosso K, Blyden D. Feasibility of common carotid artery point of care ultrasound in cardiac output measurements compared to invasive methods. J Ultrasound 2015; 18 : $127-133$
[9] Gil AL, Neto GR, Sousa MS, Dias I, Vianna J, Nunes RA, Novaes JS. Effect of strength training with blood flow restriction on muscle power and submaximal strength in eumenorrheic women. Clin Physiol Funct Imaging 2017; 37: 221-228

[10] Guilhem G, Cornu C, Guevel A. Neuromuscular and muscle-tendon system adaptations to isotonic and isokinetic eccentric exercise. Ann Phys Rehabil Med 2010; 53: 319-341

[11] Harriss DJ, Macsween A, Atkinson G. Standards for ethics in sport and exercise science research: 2018 Update. Int J Sports Med 2017; 38: 1126-1131

[12] Hashimoto M, Akishita M, Eto M, Ishikawa M, Kozaki K, Toba K, Sagara Y, Taketani Y, Orimo H, Ouchi Y. Modulation of endothelium-dependent flow-mediated dilatation of the brachial artery by sex and menstrual cycle. Circulation 1995; 92: 3431-3435

[13] Herzog W. Mechanisms of enhanced force production in lengthening (eccentric) muscle contractions. J Appl Physiol 2014; 116: 1407-1417

[14] Hicks AL, Kent-Braun J, Ditor DS. Sex differences in human skeletal muscle fatigue. Exerc Sport Sci Rev 2001; 29: 109-112

[15] Hill E, Housh T, Smith C, Cochrane K, Jenkins N, Schmidt R, Johnson G. Muscle- and mode-specific responses of the forearm flexors in women as a result of fatiguing, concentric muscle actions. Int J Phys Educ Sports Health 2016; 3: 431-438

[16] Hill E, Housh T, Smith C, Schmidt R, Johnson G. Muscle- and mode-specific responses of the forearm flexors to fatiguing, concentric muscle actions. Sports (Basel) 2016; 4: 47

[17] Hill EC, Housh TJ, Keller JL, Smith CM, Schmidt RJ, Johnson GO. Genderand muscle- specific responses during fatiguing exercise. J Strength Cond Res 2018; 32: 1471-1478

[18] Hill EC, Housh T], Smith CM, Cochrane KC, Jenkins NDM, Cramer JT, Schmidt RJ, Johnson GO. Effect of sex on torque, recovery, EMG, and MMG responses to fatigue. J Musculoskelet Neuronal Interact 2016; 16: $310-317$

[19] Hill EC, Housh TJ, Smith CM, Keller JL, Schmidt RJ, Johnson GO. The contributions of arterial cross-sectional area and time-averaged flow velocity to arterial blood flow. J Med Ultrasound 2018, Epub doi:10.4103/JMU.JMU_20_18

[20] Hunter SK. Sex differences in human fatigability: Mechanisms and insight to physiological responses. Acta Physiol (Oxf) 2014; 210: 768-789

[21] Hunter SK. Sex differences in fatigability of dynamic contractions. Exp Physiol 2016; 101: 250-255

[22] Hunter SK, Butler JE, Todd G, Gandevia SC, Taylor JL. Supraspinal fatigue does not explain the sex difference in muscle fatigue of maximal contractions. J Appl Physiol 2006; 101: 1036-1044

[23] Hunter SK, Critchlow A, Shin IS, Enoka RM. Fatigability of the elbow flexor muscles for a sustained submaximal contraction is similar in men and women matched for strength. J Appl Physiol 2004; 96: 195-202

[24] Hunter SK, Enoka RM. Sex differences in the fatigability of arm muscles depends on absolute force during isometric contractions. J Appl Physiol 2001; 91: 2686-2694

[25] Jackson AS, Pollock ML. Practical assessment of body composition. Phys Sportsmed 1985; 13: 76-90

[26] Kang T, Seo Y, Park J, Dong E, Seo B, Han D. The effects of elbow joint angle change on the elbow flexor muscle activation in pulley with weight exercise. J Phys Ther Sci 2013; 25: 1133-1136

[27] Kent-Braun JA, Ng AV, Doyle JW, Towse TF. Human skeletal muscle responses vary with age and gender during fatigue due to incremental isometric exercise. J Appl Physiol 2002; 93: 1813-1823

[28] Krejza J, Mariak Z, Huba M, Wolczynski S, Lewko J. Effect of endogenous estrogen on blood flow through carotid arteries. Stroke 2001; 32: $30-36$ 
[29] Lee A, Baxter ], Eischer C, Gage M, Hunter S, Yoon T. Sex differences in neuromuscular function after repeated eccentric contractions of the knee extensor muscles. Eur J Appl Physiol 2017; 117: 1119-1130

[30] Levenson J, Pessana F, Gariepy J, Armentano R, Simon A. Gender differences in wall shear-mediated brachial artery vasoconstriction and vasodilation. J Am Coll Cardiol 2001; 38: 1668-1674

[31] Mattacola CG, Perrin DH, Gansneder BM, Allen JD, Mickey CA. A comparison of visual analog and graphic rating scales for assessing pain following delayed onset muscle soreness. J Sport Rehab 1997; 6: 38-46

[32] Maughan RJ, Harmon M, Leiper JB, Sale D, Delman A. Endurance capacity of untrained males and females in isometric and dynamic muscular contractions. Eur ] Appl Physiol Occup Physiol 1986; 55 : 395-400

[33] McHugh MP, Tyler TF, Greenberg SC, Gleim GW. Differences in activation patterns between eccentric and concentric quadriceps contractions. J Sports Sci 2002; 20: 83-91

[34] Nardone A, Romano C, Schieppati M. Selective recruitment of high-threshold human motor units during voluntary isotonic lengthening of active muscles. J Physiol 1989; 409: 451-471

[35] Nichols AW, Hetzler RK, Villanueva RJ, Stickley CD, Kimura IF. Effects of combination oral contraceptives on strength development in women athletes. J Strength Cond Res 2008; 22: 1625-1632

[36] Orizio C, Gobbo M, Diemont B, Esposito F, Veicsteinas A. The surface mechanomyogram as a tool to describe the influence of fatigue on biceps brachii motor unit activation strategy. Historical basis and novel evidence. Eur J Appl Physiol 2003; 90: 326-336

[37] Osada T, Murase N, Kime R, Katsumura T, Rådegran G. Blood flow dynamics in the limb conduit artery during dynamic knee extensor exercise assessed by continuous Doppler ultrasound measurements. J Phys Fit Sports Med 2014; 3: 409-421

[38] Osada T, Radegran G. Alterations in the blood velocity profile influence the blood flow response during muscle contractions and relaxations. J Physiol Sci 2006; 56: 195-203

[39] Parker BA, Smithmyer SL, Pelberg JA, Mishkin AD, Herr MD, Proctor DN. Sex differences in leg vasodilation during graded knee extensor exercise in young adults. J Appl Physiol 2007; 103: 1583-1591

[40] Power GA, Dalton BH, Rice CL, Vandervoort AA. Delayed recovery of velocity- dependent power loss following eccentric actions of the ankle dorsiflexors. J Appl Physiol 2010; 109: 669-676

[41] Power GA, Dalton BH, Rice CL, Vandervoort AA. Peak power is reduced following lengthening contractions despite a maintenance of shortening velocity. Appl Physiol Nutr Metab 2013; 38: 1196-1205

[42] Prasartwuth O, Allen T], Butler JE, Gandevia SC, Taylor JL. Lengthdependent changes in voluntary activation, maximum voluntary torque and twitch responses after eccentric damage in humans. J Physiol 2006; 571: 243-252
[43] Russ DW, Kent-Braun JA. Sex differences in human skeletal muscle fatigue are eliminated under ischemic conditions. J Appl Physiol 2003; 94: 2414-2422

[44] Sakamaki M, Yasuda T, Abe T. Comparison of low-intensity blood flow-restricted training-induced muscular hypertrophy in eumenorrheic women in the follicular phase and luteal phase and age-matched men. Clin Physiol Funct Imaging 2012; 32: 185-191

[45] Sarwar R, Niclos BB, Rutherford OM. Changes in muscle strength, relaxation rate and fatiguability during the human menstrual cycle. J Physiol 1996; 493: 267-272

[46] Sayers SP, Clarkson PM. Force recovery after eccentric exercise in males and females. Eur J Appl Physiol 2001; 84: 122-126

[47] Senefeld J, Pereira HM, Elliott N, Yoon T, Hunter SK. Sex differences in mechanisms of recovery after isometric and dynamic fatiguing tasks. Med Sci Sports Exerc 2018; 50: 1070-1083

[48] Senefeld J, Yoon T, Bement MH, Hunter SK. Fatigue and recovery from dynamic contractions in men and women differ for arm and leg muscles. Muscle Nerve 2013; 48: 436-439

[49] Sewright KA, Hubal M], Kearns A, Holbrook MT, Clarkson PM. Sex differences in response to maximal eccentric exercise. Med Sci Sports Exerc 2008; 40: 242-251

[50] Solomonow M, Baratta R, Zhou BH, D’Ambrosia R. Electromyogram coactivation patterns of the elbow antagonist muscles during slow isokinetic movement. Exp Neurol 1988; 100: 470-477

[51] Sung E, Han A, Hinrichs T, Vorgerd M, Manchado C, Platen P. Effects of follicular versus luteal phase-based strength training in young women. SpringerPlus 2014; 3: 668

[52] Wakeling JM, Uehli K, Rozitis Al. Muscle fibre recruitment can respond to the mechanics of the muscle contraction. J R Soc Interface 2006; 3: 533-544

[53] Weir JP. Quantifying test-retest reliability using the intraclass correlation coefficient and the SEM. J Strength Cond Res 2005; 19 : 231-240

[54] Wikstrom-Frisen L, Boraxbekk C], Henriksson-Larsen K. Effects on power, strength and lean body mass of menstrual/oral contraceptive cycle based resistance training. J Sport Med Phys Fitness 2017; 57: 43-52

[55] Williams MR, Westerman RA, Kingwell BA, Paige ], Blombery PA, Sudhir K, Komesaroff PA. Variations in endothelial function and arterial compliance during the menstrual cycle. J Clin Endocrin Metab 2001; 86: 5389-5395

[56] Yoon T, Doyel R, Widule C, Hunter SK. Sex differences with aging in the fatigability of dynamic contractions. Exp Gerontol 2015; 70: $1-10$ 